\title{
Effects of Aperture Size on $Q$ factor and Shielding Effectiveness of a Cubic Resonator
}

\author{
Stefan Parr ${ }^{1}$, Stephan Chromy ${ }^{1}$, Stefan Dickmann ${ }^{1}$, and Martin Schaarschmidt ${ }^{2}$ \\ ${ }^{1}$ Faculty of Electrical Engineering, Helmut-Schmidt-University/University of the Federal Armed Forces Hamburg, \\ Hamburg, Germany \\ ${ }^{2}$ Bundeswehr Research Institute for Protective Technologies and NBC Protection (WIS) Munster, Munster, Germany \\ Correspondence to: Stefan Parr (stefan.parr@gmx.de)
}

Received: 13 December 2016 - Revised: 3 May 2017 - Accepted: 6 July 2017 - Published: 21 September 2017

\begin{abstract}
The EMC properties of a cubic metallic shield are highly affected by its resonances. At the resonant frequencies, the shielding effectiveness (SE) collapses, which results in high field strengths inside the cavity. This can cause failure or even breakdown of electronic devices inside the shield. The resonant behaviour is mainly determined by the quality or $Q$ factor of the shield. In this paper, the effects of the aperture size on the $Q$ factor and the SE of an electrically large, cubic shield are analysed. At first, a method is developed in order to determine the $Q$ factor based on the resonance behaviour of the shield in time domain. Only the first resonance of the shield is considered therefore. The results are evaluated for different aperture diameters and compared with theory for the $Q$ factor. The dominant coupling mechanism of electromagnetic energy into the shield is thus identified.

Then the effect of aperture size on the SE is analysed. The excitation of resonances is very probable if the interfering signal is an ultrawideband (UWB) pulse, which constitutes a typical intentional electromagnetic interference (IEMI) scenario. Therefore, the relation between aperture size and SE is analysed using the theory of the transient SE for a broadband signal with a constant spectral density distribution. The results show, that a "worst case" aperture size exists, where the SE has its minimum.
\end{abstract}

\section{Introduction}

\subsection{The quality factor $Q$}

The $Q$ factor of an electrically large, lossy cavity has been calculated by Hill et al. (1994), assuming a uniform energy density throughout the cavity. On the one hand, this theory is only an approximation, as cavity resonances and the exact interior field strength distribution are not considered. On the other hand, it allows to consider the energy absorption by wall losses, by absorption inside the cavity and by antenna measurements. Thus it is applicable for real life setups like shields containing electronic equipment. Also, a relation of the $Q$ factor and the SE via a power balance approach has been given. The calculation of SE via this method has been done before by Armstrong et al. (2011) and Xu et al. (2015). An advantage of this method is the possibility to calculate SE of large enclosures, that cannot be irradiated from outside. The determination of the $Q$ factor of a cavity is possible by measuring the width of autocorrelation, which is called the WA-method by Armstrong et al. (2011). Another method using a reverberation chamber and a spectrum analyser has been developed by Krauthäuser and Nitsch (2002). In this paper, $Q$ is extracted from the resonance behaviour in time domain, observing the decay of the field strength inside the shield. In the following, a short introduction to the $Q$ factor theory by Hill et al. (1994) is given.

The $Q$ factor of a resonator depends on the ratio of the energy stored in the oscillating system $W(t)$, and the energy loss during one period $T$. It is defined as:

$Q=2 \pi \frac{W(t)}{W(t)-W(t+T)}$.

The energy stored in the system decays exponentially according to:

$W(t)=W_{0} \mathrm{e}^{-t / \tau}$, 
with $W_{0}=W(t=0)$ and $\tau$ : characteristic time constant. The $Q$ factor can be written as

$Q=\tau \omega$,

where $\omega$ is the angular frequency. Four different loss mechanisms determine the overall quality factor:

- currents in the shield's walls: $Q_{\text {surf (“surface") }}$

- absorbing materials inside the shield: $Q_{\text {ACS }}$ ("Aperture Cross Section")

- aperture losses: $Q_{\mathrm{TCS}}$ (“Transmission Cross Section”)

- losses of the antenna used for field measurement inside the shield: $Q_{\text {ant }}$ ("antenna").

Thus, $Q$ can be written as

$\frac{1}{Q}=\frac{1}{Q_{\text {surf }}}+\frac{1}{Q_{\mathrm{ACS}}}+\frac{1}{Q_{\mathrm{TCS}}}+\frac{1}{Q_{\mathrm{ant}}}$.

This implies, that the overall quality factor $Q$ is dominated by the lowest partial $Q$. In this paper, the effect of the aperture size on the $Q$ factor is investigated. The partial $Q$ factor $Q_{\text {TCS }}$ for a resonator with circular aperture can be written as:

$Q_{\mathrm{TCS}}=\frac{4 \pi V_{\text {shield }}}{\lambda \sigma_{\mathrm{TCS}}}$,

where $V_{\text {shield }}$ is the volume of the shield, $\lambda$ the wavelength and $\sigma_{\mathrm{TCS}}$ the transmission cross section, which is given in Hill et al. (1994) for circular apertures. If the aperture is electrically small and the direction of the incident transverse electromagnetic (TEM) wave is perpendicular to the front side of the shield as in fig. $1, \sigma_{\text {TCS }}$ can be written as:

$\sigma_{\mathrm{TCS}}=\frac{k^{4} d^{6}}{27 \pi}$,

where $k$ is the wavenumber and $d$ the diameter of the circular aperture. For an electrically large aperture,

$\sigma_{\mathrm{TCS}}=\pi\left(\frac{d}{2}\right)^{2}$

which is equal to the area of the aperture. At a diameter of

$d_{\lim } \approx 0.41 \lambda$

the aperture is in resonance. For a resonant aperture, there is no approximation of the transmission cross section $\sigma_{\mathrm{TCS}}$ available. However, the circular aperture does not have strong resonances according to Hill et al. (1994).

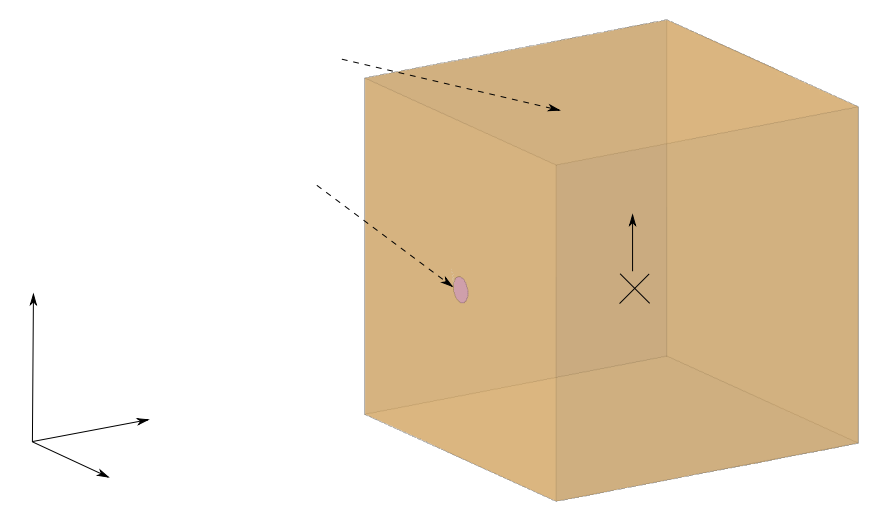

Figure 1. Cubic, metallic shield with a circular aperture and incident TEM wave.

\subsection{Shielding Effectiveness}

In Sect. 3, the effect of the aperture size on SE is evaluated for a resonator model shown in Fig. 1. It consists of a cubic resonator with a circular aperture of variable dimension. Intuitively, there are two different opposite effects occurring. On one hand, for small aperture dimensions, the field coupling inside the shield is small, but due to the high quality factor, strong resonance effects can be the reason for high peak field strengths for continuous wave (cw) fields. On the other hand, for large apertures, the field coupling inside the shield is strong, even though resonances are less prevailing.

The electric SE at the point $q$ is defined in the frequency domain as:

$\left.\mathrm{SE}_{\text {el }}\right|_{q}=20 \log _{10} \frac{\left|\boldsymbol{E}^{\text {unshield }}\right|}{\left|\boldsymbol{E}^{\text {shield }}\right|} \mathrm{dB}$.

It is difficult to evaluate the EMC properties of a shielded room with regard to a broadband electric pulse as interfering signal. However, such pulses with a short rise time constitute a typical intentional electromagnetic environment (IEME) according to Giri and Tesche (2004). UWB pulses can have spectral components up to several $\mathrm{GHz}$, which makes the excitation of cavity resonances probable. In order to consider the spectral density distribution of the interfering incident signal, another definition of the SE is available, the transient shielding effectiveness $\left.\mathrm{SE}_{\mathrm{t}}\right|_{q}$ according to Klinkenbusch (2005):

$$
\begin{aligned}
& \left.\mathrm{SE}_{\mathrm{t}}\right|_{q}=10 \log _{10} \\
& \frac{2 \int_{0}^{\infty}\left|S^{\text {inc }}\right|^{2} \omega \mathrm{d} \omega}{\int_{0}^{\infty}\left|S^{\text {inc }}\right|^{2}\left[\frac{\left|\boldsymbol{E}^{\text {shield }}\right|_{q}^{2}}{\left|\boldsymbol{E}^{\text {unshield }}\right|_{q}^{2}}+\frac{\left|\boldsymbol{H}^{\text {shield }}\right|_{q}^{2}}{\left|\boldsymbol{H}^{\text {unshield }}\right|_{q}^{2}}\right] \omega \mathrm{d} \omega} \mathrm{dB},
\end{aligned}
$$

where $q$ is the point of measurement inside the shield, $S^{\text {inc }}$ the spectral density distribution of the incoming poynting vector, $\boldsymbol{E}$ and $\boldsymbol{H}$ the electric and magnetic field vectors. This 
definition is used to evaluate the transient SE of the resonator model. As an interfering signal, a broadband pulse is assumed.

\section{Effect of the Aperture Size on the $Q$ factor}

\subsection{Measurement setup}

The effect of the aperture size on the $Q$ factor is analysed for the resonator model shown in Fig. 1. As the shield is cubic, different modes are occurring at the same frequency, which makes it a degenerate resonator. This intensifies the resonant behaviour. The cube has an edge length of app. $40 \mathrm{~cm}$ and a variable diameter of the circular aperture. For the measurement of its $Q$ factor, it is irradiated with a pulse modulated continuous wave (cw) signal. The output voltage of the signal generator can be written as:

$u_{\text {signal }}(t)= \begin{cases}U_{0} \cdot \sin (\omega t), & 0<t \leq T_{\text {pulse }} \\ 0, & T_{\text {pulse }}<t \leq T_{\text {end }} .\end{cases}$

Here, $U_{0}$ is the amplitude, $T_{\text {pulse }}$ the pulse width $(10 \mu \mathrm{s})$, and $T_{\text {end }}$ the length of the signal. The frequency of the oscillation is chosen to be $527 \mathrm{MHz}$, which corresponds to the first resonant frequency of the shield. The output voltage $u_{\text {signal }}(t)$ is amplified and irradiated via a log-periodic antenna inside an anechoic chamber. The electric field $\left.\boldsymbol{E}^{\text {shield }}\right|_{q}$ is measured in the middle of the shield with a D-DOT sensor. In this case this is sufficient, as the considered resonance at $527 \mathrm{MHz}$ has an anti-node of the electric field there. The D-DOT sensor has an output voltage of

$u_{\mathrm{D}-\mathrm{DOT}}(t)=R A_{\mathrm{eq}} \frac{\mathrm{d} D}{\mathrm{~d} t}$,

where $R$ is the load impedance of the sensor, $A_{\text {eq }}$ the equivalent sensor area and $D$ the electric displacement field.

\subsection{Calculation of the $Q$ factor}

The output voltage of the D-DOT sensor $u_{\mathrm{D}-\mathrm{DOT}}(t)$ and the pulse duration are shown for an aperture diameter of $6 \mathrm{~cm}$ in Fig. 2. The oscillation inside the shield decays exponentially

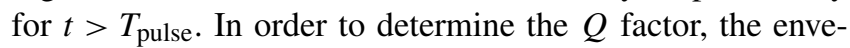
lope $u_{\text {env }}(t)$ of the oscillation is calculated via the Hilbert transform:

$u_{\mathrm{env}}(t)=\mid H\left(u_{\mathrm{D}-\mathrm{DOT}}(t) \mid\right.$.

Then the discrete values of the envelope are fitted with an exponential function. The result of the fitting is shown in Fig. 3. From the fitting parameters, $Q$ can be calculated from the time constant $\tau$ with Eq. (3). The $Q$ factor is thus determined for aperture diameters of 3,6,12,16 and $20 \mathrm{~cm}$. It is valid only for the first resonance at $527 \mathrm{MHz}$, which has been excited. The results are depicted in Fig. 4. Additionally, the theoretical approximations for $Q_{\mathrm{TCS}}$ for electric small

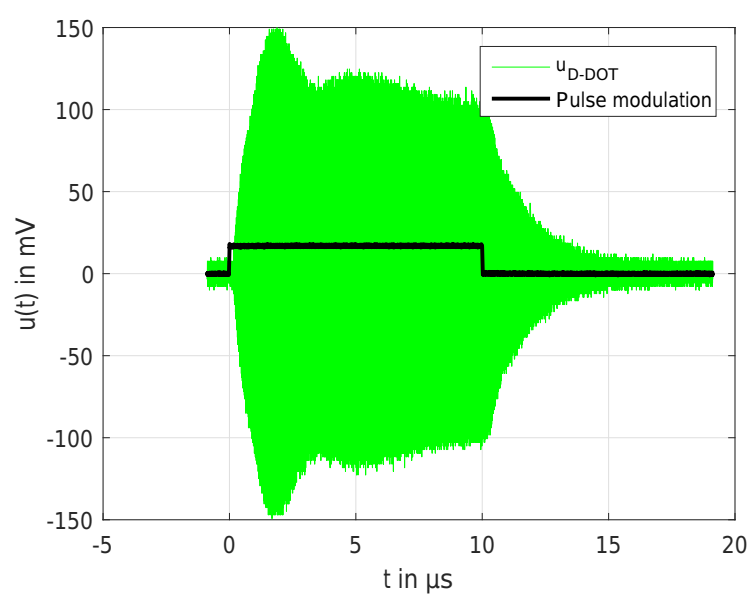

Figure 2. Resonant behaviour of the shield for $d=6 \mathrm{~cm}$

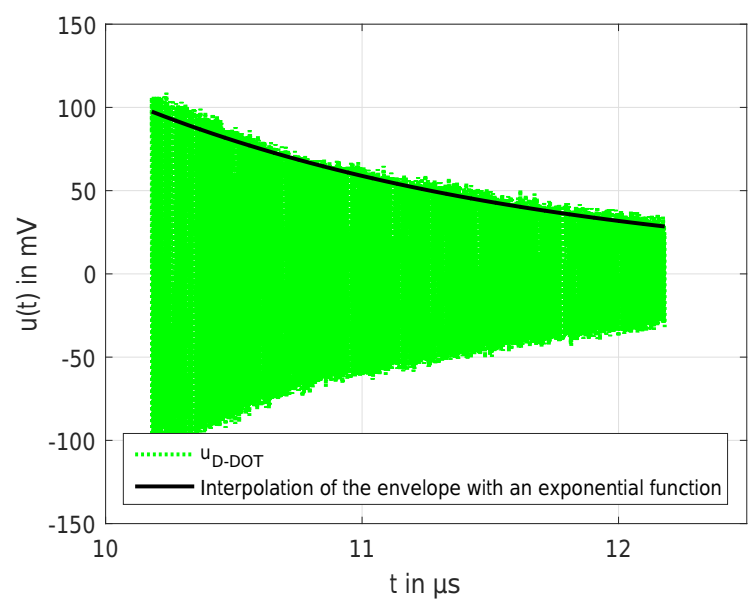

Figure 3. Exponential decay of the oscillation and curve fitting of the envelope $u_{\text {env }}(t)$ for $d=6 \mathrm{~cm}$.

and large apertures and $d_{\text {lim }}$ according to Eqs. (5), (6), (7) and (8) are shown. It can be seen that for small aperture diameters, the differences between the measured $Q$ factor and $Q_{\text {TCS }}$ are quiet large. This means, that the aperture losses are not the dominant loss mechanism for small aperture diameters, but losses by the field measurement or current losses in the shield's walls or EMC gaskets. For diameters greater than $10 \mathrm{~cm}$, the aperture losses are the dominating factor.

The $Q$ factor theory according to Hill et al. (1994) does not consider the exact field distribution inside the shield due to cavity resonances, but the energy density throughout the cavity is assumed to be uniform. Yet the analysed cubic resonator shows strong resonant behaviour. That explains the overall mismatch between measurement results and theory, even when the aperture effect prevails for aperture diameters larger than $12 \mathrm{~cm}$. For $d=24 \mathrm{~cm}$, the difference between measurement and theory is at its maximum. This is caused by an additional error: the aperture resonance at $d_{\text {lim }}$ is not considered in the approximation for $Q_{\mathrm{TCS}}$. 


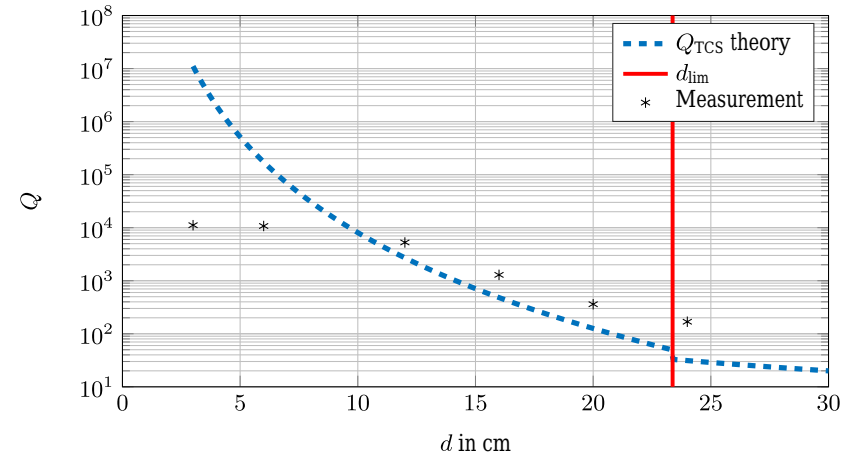

Figure 4. Measured $Q$ factor of the first resonance at $527 \mathrm{MHz}$ for different aperture diameters.

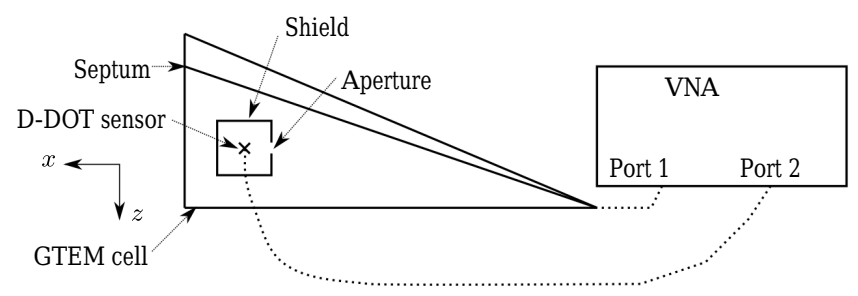

Figure 5. Measurement setup for the calculation of the transient SE for different aperture sizes.

\section{Effect of Aperture Size on SE}

Now, the effect of the aperture diameter $d$ on the SE of the resonator model shown in Fig. 1 is analysed. The measurement setup for the calculation of the transient SE is shown in Fig. 5. It consists of a vector network analyser (VNA), which is connected to the GTEM cell and the D-DOT sensor via a coaxial cable. The cable is guided into the GTEM cell and the shield via shielded adaptors for panel mounting. The D-DOT sensor is connected directly to the adaptors on the inside of the shield. So in contrary to the metallic D-DOT sensor, the cables do not influence the resonant behaviour of the shield. The chosen measurement bandwidth of the VNA is $100 \mathrm{kHz}$ with a step width of $95 \mathrm{kHz}$ in order to ensure a complete coverage of the spectrum in the frequency range from $100 \mathrm{MHz}$ up to $2 \mathrm{GHz}$. The $S$ parameters for the unshielded and shielded case for aperture diameters of 12 and $24 \mathrm{~cm}$ are shown exemplarily in Fig. 6. The values are filtered with a rectangular window function with a length of ten in order to improve visibility. The different values for the bandwidth at the first resonance peak at $527 \mathrm{MHz}$ are visible, according to the different $Q$ factors. The maximum coupling occurs at this resonance. As the spectral density distribution of UWB signals usually decreases with frequency, the highest field coupling is to be expected at this resonance. Additionally, in Fig. 7, the electric SE in point $q$ according to

$\left.\mathrm{SE}_{\mathrm{el}}\right|_{q}=20 \log _{10} \frac{\left|S_{21}^{\text {unshield }}\right|_{q}}{\left|S_{21}^{\text {shield }}\right|_{q}} \mathrm{~dB}$

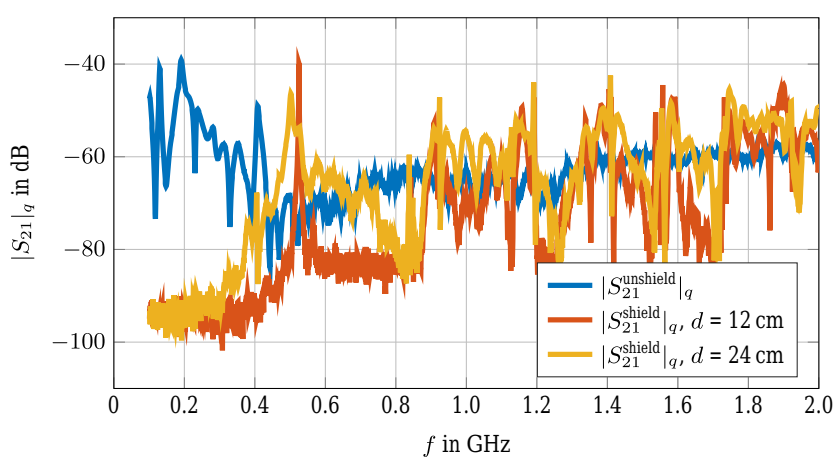

Figure 6. $S_{21}$ parameter in the unshielded and shielded case for different aperture diameters.

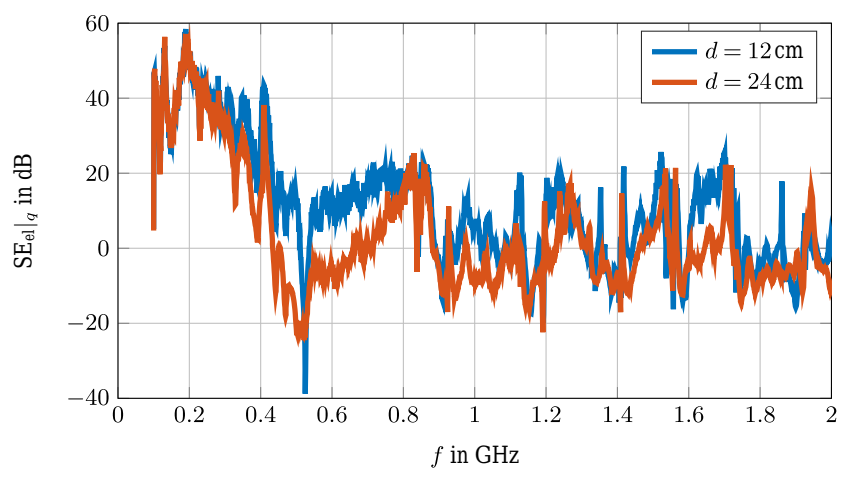

Figure 7. Electric SE in point $q$ for aperture diameters of 12 and $24 \mathrm{~cm}$.

is shown for aperture diameters of 12 and $24 \mathrm{~cm}$.

The incident wave $S^{\text {inc }}$ for the calculation of the transient SE is supposed to be a Dirac delta function with a constant spectral density distribution. As UWB signals have frequency components up to several $\mathrm{GHz}$, this is not an unrealistic assumption in the analysed frequency region up to $2 \mathrm{GHz}$. Only the $z$-component of the electric field is measured by the D-DOT sensor. Therefore, the definition for $\left.\mathrm{SE}_{\mathrm{t}}\right|_{q}$ (Eq. 10) is adapted here to the electric transient SE

$$
\left.\mathrm{SE}_{\mathrm{el}, \mathrm{t}}\right|_{q}=10 \log _{10} \frac{\omega_{2}^{2}-\omega_{1}^{2}}{2 \int_{\omega_{1}}^{\omega_{2}} \frac{\left|S_{21}^{\text {shield }}\right|_{q}^{2}}{\left|S_{21}^{\text {unshield }}\right|_{q}^{2}} \omega \mathrm{d} \omega} \mathrm{dB},
$$

which only considers the electric field. The measurement results for $\left.\mathrm{SE}_{\mathrm{el}, \mathrm{t}}\right|_{q}$ for different aperture diameters are shown in Fig. 8 together with a spline interpolation. It can be seen that for $d=\left.16 \mathrm{~cm} \mathrm{SE} \mathrm{el}_{\mathrm{t}, \mathrm{t}}\right|_{q}$ has a minimum. This aperture diameter constitutes the "worst-case" aperture. Intuitively, that can be explained by the two opposite effects: For small aperture dimensions strong resonance effects cause high field strengths at the resonant frequencies. For big apertures, the field coupling inside the shield is naturally bigger, but the resonance effects are small. 


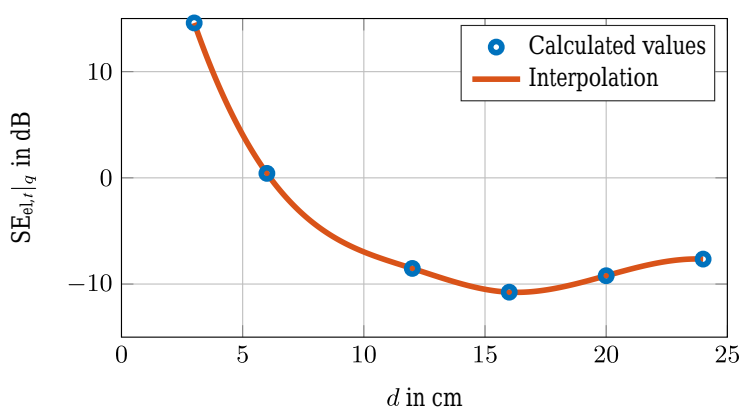

Figure 8. Electric transient SE for different aperture diameters.

This result cannot be generalized, for the measured field strength inside the shield depends on various variables: the VNA settings, the shield set-up and the measurement set-up. Not only the aperture size, but also the position of the D-DOT sensor inside the shielded volume as well as the incident direction and polarisation of the exciting field will drastically change the measured SE. To overcome these issues, several methods have been developed in the past, to get an average SE for different measurement positions and different incident directions and polarisations, like the "nested reverberation chamber technique" in Holloway et al. (2008), a dual vibrating reverberation chamber by Schipper and Leferink (2015) and a spatial averaging by Parr et al. (2014).

\section{Conclusions}

In this paper, the effects of aperture size on the $Q$ factor of a cubic resonator have been investigated. As a shield model, a cubic metallic cavity with an edge length of $40 \mathrm{~cm}$ and a circular aperture has been used. A new method has been developed that extracts the $Q$ factor from the resonant behaviour in time domain. Therefore, only the first resonance of the shield has been considered. The results of the measurement have been compared with the theory of the aperture $Q$ factor. This has shown that the field coupling inside the shield via the aperture is dominating for diameters exceeding $10 \mathrm{~cm}$. The used theory for the $Q$ factor of a resonator does not consider the exact field distribution at the first resonance, but the energy density throughout the cavity is assumed to be uniform. That is why the results of measurement and theory do not match exactly.

Also, the effect of aperture size on SE has been analysed. In order to consider the spectral density distribution of a broadband interfering signal, the concept of transient SE has been used. The field coupling into the shield has been measured inside the GTEM cell with a VNA for different aperture diameters. The results show, that the highest coupling occurs at the first resonant frequency of the shield. For the used shield, measurement and VNA set-up, the "worst-case" aperture diameter with a minimal $\mathrm{SE}_{\mathrm{el}, \mathrm{t}} \mathrm{l}_{q}$ is at $16 \mathrm{~cm}$. For the measurement of the shielded field strength, only one field component inside the shield at one point has been considered. Therefore, the higher resonances, especially those with a node in the middle of the shield, are not considered equally. Further work using methods to overcome these issues has to be done in order to make general statements.

Data availability. The data is available upon request from the author.

Competing interests. The authors declare that they have no conflict of interest.

Edited by: Frank Gronwald

Reviewed by: Sven Battermann, Mathias Magdowski, and one anonymous referee

\section{References}

Armstrong, R., Marvin, A., and Dawson, J.: An experimental investigation of the use of Q-factor to determine the shielding effectiveness of electrically large equipment enclosures with apertures, in: EMC Europe 2011 York, 148-152, 2011.

Giri, D. and Tesche, F.: Classification of intentional electromagnetic environments (IEME), IEEE T. Electromagn. C. 46, 322-328, https://doi.org/10.1109/TEMC.2004.831819, 2004.

Hill, D., Ma, M., Ondrejka, A., Riddle, B., Crawford, M., and Johnk, R.: Aperture excitation of electrically large, lossy cavities, IEEE T. Electromagn. C., 36, 169-178, https://doi.org/10.1109/15.305461, 1994.

Holloway, C., Hill, D., Sandroni, M., Ladbury, J., Coder, J., Koepke, G., Marvin, A., and He, Y.: Use of Reverberation Chambers to Determine the Shielding Effectiveness of Physically Small, Electrically Large Enclosures and Cavities, IEEE T. Electromagn. C., 50, 770-782, https://doi.org/10.1109/TEMC.2008.2004580, 2008.

Klinkenbusch, L.: On the shielding effectiveness of enclosures, IEEE T. Electromagn. C., 47, 589-601, https://doi.org/10.1109/TEMC.2005.853162, 2005.

Krauthäuser, H. G. and Nitsch, J.: Transient Fields in Mode-Stirred Chambers, in: XXVIIth General Assembly of the International Union of Radio Science, URSI 2002, 2002.

Parr, S., Karcoon, H., Dickmann, S., and Rambousky, R.: Verbesserung der Schirmeffektivität eines metallisch geschirmten Raums an den Resonanzfrequenzen mithilfe von Absorbermaterial, in: EMV - Internationale Fachmesse und Kongress für Elektromagnetische Verträglichkeit, 2014.

Schipper, H. and Leferink, F.: Shielding effectiveness measurements of materials and enclosures using a dual vibrating intrinsic reverberation chamber, in: 2015 IEEE International Symposium on Electromagnetic Compatibility (EMC), 23-28, https://doi.org/10.1109/ISEMC.2015.7256126, 2015.

Xu, Q., Huang, Y., Zhu, X., Xing, L., Tian, Z., and Song, C.: Shielding Effectiveness Measurement of an Electrically Large Enclosure Using One Antenna, IEEE T. Electromagn. C., 57, 14661471, https://doi.org/10.1109/TEMC.2015.2477455, 2015. 